Pacific Journal of Mathematics

EXTENSIONS OF REPRESENTATIONS OF LIE ALGEBRAS 


\title{
EXTENSIONS OF REPRESENTATIONS OF LIE ALGEBRAS
}

\author{
JOHN GERARD RYAN
}

\begin{abstract}
Let $\phi: L_{1} \rightarrow L_{2}$ be a morphism of finite-dimensional Lie algebras over a field of characteristic zero. Our problem is this: given a finite-dimensional $L_{1}$-module, $V$ say, when does $V$ embed as a sub $L_{1}$-module of some finite-dimensional $L_{2}$-module? The problem clearly reduces to the case in which $\phi$ is injective. We provide here (Thm. 3.6) a solution in two separate cases: (i) under the assumption that $\phi$ maps the radical of $L_{1}$ into the radical of $L_{2}$, or (ii) under the assumption that $L_{1}$ is its own commutator ideal.
\end{abstract}

0. Introduction. A theorem of Bialynicki-Birula, Hochschild, and Mostow ([1, Thm. 1]) gives conditions for a finite-dimensional module for a subgroup of an algebraic group to embed as a submodule into a finite-dimensional module for the whole group. It is with a modification of this result that we obtain criteria for modules of Lie algebras.

Throughout this paper, $k$ will denote a field of characteristic zero, and $K$ will be an algebraic closure of $k$. For a Lie algebra $L$ over $k, U(L)$ will denote the universal enveloping algebra of $L ; H(L)$ will denote the Hopf algebra of representative functions associated with $L$. All of our Lie algebras, modules, and representations are taken to be finite-dimensional unless otherwise specified. We will regard a module for a Lie algebra $L$ as also a left $U(L)$-module or as a right $H(L)$-comodule, and vice versa.

The author wishes to thank Professor G. Hochschild for his generous and invaluable assistance in the suggestion of the topic and in the writing of the doctoral dissertation on which this paper is based.

\section{Reduction of the problem to representative functions.}

Definition. Let $\phi: H_{1} \rightarrow H_{2}$ be a morphism of coalgebras over $k . \phi$ induces an $H_{2}$-comodule structure on any $H_{1}$-comodule $\psi: V \rightarrow V \otimes H_{1}$ by following up $\psi$ with $(i \otimes \phi)$, where $i$ is the identity map. We say that an $H_{2}$-comodule $\xi: U \rightarrow U \otimes H_{2}$ is extendable to $H_{1}$ if there is an $H_{1}$-comodule $\psi: V \rightarrow V \otimes H_{1}$ and a linear injection $j: U \hookrightarrow V$ such that $(j \otimes i) \circ \xi=(i \otimes \phi) \circ \psi \circ j$. 
Clearly, a necessary condition for $\xi$ to be extendable is that $\xi(U)$ should be contained in $U \otimes \phi\left(H_{1}\right)$. We say that $\phi$ is manageable if, for all $\mathrm{H}_{2}$-comodules $\mathrm{U}$, the above condition is also sufficient.

Note. $[1, \S 6]$ contains examples of morphisms $\phi$ that are not manageable.

We remark that, if $H$ is a bialgebra, then the multiplication map on $H$ enables us to construct an $H$-comodule structure on the tensor product of two comodules. If $H$ is commutative, then the $H$-comodule structure on the tensor algebra $\otimes V$ of an $H$-comodule $V$ factors to give an $H$-comodule structure on each homogeneous component of the exterior algebra $\wedge V$ built on $V$. Finally, we note that, if $H$ is a Hopf algebra, then the antipode map on $H$ enables us to construct an $H$-comodule structure on the linear dual $V^{o}$ of an $H$-comodule $V$.

The following is a generalization of [1, Thm. 1].

LemMa 1.1. Let $\phi: H_{1} \rightarrow H_{2}$ be a morphism of commutative Hopf algebras over $k$. Then $\phi$ is manageable if and only if, for every one-dimensional $\mathrm{H}_{2}$-comodule that is extendable to $H_{1}$, the dual comodule $V^{\mathrm{o}}$ is also extendable to $H_{1}$.

Proof. The necessity of the condition is clear. Now, suppose that the condition on one-dimensional $\mathrm{H}_{2}$-comodules is satisfied. Let $\xi: U \rightarrow U \otimes$ $\mathrm{H}_{2}$ be an $\mathrm{H}_{2}$-comodule, and let us assume that $\xi(U)$ is contained in $U \otimes \phi\left(H_{1}\right)$. Then, $U$ is isomorphic with a subcomodule of the direct sum of finitely many copies of $\phi\left(H_{1}\right)$, where the (locally finite-dimensional) $H_{2}$-comodule structure on $\phi\left(H_{1}\right)$ is given by the restriction of the comultiplication of $\mathrm{H}_{2}$.

If we take inverse images under the map that sends $H_{1} \oplus \cdots \oplus H_{1}$ to $\phi\left(H_{1}\right) \oplus \cdots \oplus \phi\left(H_{1}\right)$, we can choose a finite-dimensional sub $H_{1}$-comodule $Z$ of $H_{1} \oplus \cdots \oplus H_{1}$ and a sub $H_{2}$-comodule $X$ of $Z$ that maps onto $U$ (in $\phi\left(H_{1}\right) \oplus \cdots \oplus \phi\left(H_{1}\right)$ ) with kernel $Y$, say. Let $n$ be the dimension of $Y$. Now, $U \otimes \wedge^{n} Y$ is an $H_{2}$-comodule and, with the identification of $U$ with $X / Y$, the multiplication of the algebra $\wedge Z$ yields an isomorphism from $U \otimes \wedge^{n} Y$ to $X\left(\wedge^{n} Y\right)$. Now, observe that $\wedge^{n} Y$ is a one-dimensional $H_{2}$-comodule that is extendable to $H_{1}$; by assumption, then, there is an $H_{1}$-comodule $V$ that contains $\left(\wedge^{n} Y\right)^{\circ}$ as a sub $H_{2}$-comodule. It is thus clear that there is an embedding of $U\left(\cong U \otimes \wedge^{n} Y \otimes\left(\wedge^{n} Y\right)^{\circ}\right)$ into the $H_{1}$-comodule $\wedge^{n+1} Z \otimes V$. 
COROLlaRY 1. If the only one-dimensional $\mathrm{H}_{2}$-comodule is the trivial comodule, then every morphism of Hopf algebras $\phi: \mathrm{H}_{1} \rightarrow \mathrm{H}_{2}$ is manageable.

COROLlaRY 2. If $H_{1}$ is a pointed Hopf algebra, that is, if the simple $H_{1}$-comodules are one-dimensional, then every $\phi: H_{1} \rightarrow H_{2}$ is manageable.

Proof. If a one-dimension $H_{2}$-comodule $U$ embeds in some $H_{1}$-comodule, then it embeds in a simple $H_{1}$-comodule, say $V$. Since $V$ is one-dimensional, the embedding of $U$ into $V$ is an $\mathrm{H}_{2}$-comodule isomorphism which yields an embedding of $U^{\circ}$ as a sub $\mathrm{H}_{2}$-comodule of the $H_{1}$-comodule $V^{\mathrm{o}}$.

At this stage, it is useful to simplify the problem by working over an algebraically closed field. This involves no loss of generality.

THEOREM 1.2. Let $\phi: L_{1} \rightarrow L_{2}$ be a morphism of Lie algebras over $K$, and let $\phi^{*}: H\left(L_{2}\right) \rightarrow H\left(L_{1}\right)$ be the corresponding map of representative functions. Suppose that either $(a) \phi$ sends the radical $R_{1}$ of $L_{1}$ into the radical $R_{2}$ of $L_{2}$, or (b) $L_{1}=\left[L_{1}, L_{1}\right]$. Then the map $\phi^{*}$ is manageable.

Proof. Condition (b) implies that the one-dimensional $L_{1}$-modules are trivial, i.e. that the one-dimensional $H\left(L_{1}\right)$-comodule are trivial. Therefore the manageability of $\phi^{*}$ in this case follows from Corollary 1 above.

Suppose now that we are in case (a). Let $U$ be a one-dimensional $L_{1}$-module that embeds in an $L_{2}$-module $V$. We can assume that $V$ is simple (consider a composition series for $V$ ). Then, in particular, $\left[L_{2}, R_{2}\right]$ annihilates $V$. By a well-known result, $V$ will be semisimple as an $R_{2}$-module and consequently, as such, is a direct sum $\oplus V_{i}$ of one-dimensional sub $R_{2}$-modules. It is clear that we can embed $U$ as a sub $R_{1}$-module into one of these $R_{2}$-modules $V_{i}$. Let $S_{1}$ be a maximal semisimple subalgebra of $L_{1}$ and let $S_{2}$ be a maximal semisimple subalgebra of $L_{2}$ that contains $\phi\left(S_{1}\right)$. Since $U$ is a one-dimensional $L_{1}$-module, it is annihilated by $S_{1}$. Since $\left[L_{1}, L_{2}\right]$ annihilates $V_{i}$, we can extend the $R_{2}$-module structure on $V_{i}$ to an $L_{2}$-module structure by making $S_{2}$ act trivially. Then the embedding of $U$ into $V_{i}$ is an embedding of $L_{1}$-modules. As we have seen in the proof of Corollary 2 above, it follows that the dual of $U$ can be embedded in the dual of $V_{l}$. In view of Lemma 1.1, this completes the proof. 


\section{An analysis of the Hopf algebra of representative functions.}

Notation. For any Lie algebra $L$, we denote by $Q(L)$ the (multiplicative) group of group-like elements of $H(L)$, and by $P(L)$ the (additive) group of primitive elements of $H(L)$. Note that there is an isomorphism of groups from $P(L)$ to $Q(L)$ given by the exponential map.

Definition. ([3], [5], [6]). Let $L$ be a Lie algebra over $K$. A subalgebra $J$ of $H(L)$ is called a basic subalgebra if the multiplication map yields an algebra isomorphism from $J \otimes K[Q(L)]$ to $H(L)$. Let $R$ denote the radical of $L$. A basic subalgebra $J$ is called a normal basic subalgebra if the semisimple part $J_{s}$ of $J$ (i.e. the subalgebra consisting of representative functions belonging to semisimple representations of $L$ ) is exactly the left $R$-annihilated part $H(L)^{R}$ of $H(L)$ and if $J$ is a left $H(L)$-comodule.

The main results on basic subalgebras that we need are that, for any Lie algebra $L$, a normal basic subalgebra of $H(L)$ always exists $([6, \mathrm{p}$. 610]), that any two normal basic subalgebras of $H(L)$ are conjugate via an automorphism of $H(L)$ of the form $\operatorname{Exp}(t(x))$ where $t$ is the left-translation map and $x$ is in $[L, R]([3$, Thm. 4.1]), that every normal basic subalgebra contains the group $P(L)$ of primitive elements and is finitely generated as an algebra ([6, Thm. 4]).

The existence and conjugacy of normal basic subalgebras implies the existence of a unique small sub Hopf algebra $B(L)$ of $H(L)$ such that $B(L)$ contains some (and hence every) normal basic subalgebra of $H(L)$. We call $B(L)$ the basic sub Hopf algebra of $H(L)$.

In the rest of this section, $L$ is a Lie algebra over $K, R$ is the radical of $L, N=[L, R]$ (this coincides with the intersection of the kernels of all semisimple representations of $L), t$ is the left translation map on $H(L)$ and $t_{r}$ the right-translation map.

Lemma 2.1. Let $H$ be a sub Hopf algebra of $H(L)$ and suppose that $H$ contains a normal basic subalgebra J. Then, the intersection of $H$ with $Q(L)$ is a set of free generators for $H$ as a J-module.

Proof. As is easy to see, it is sufficient to show that $H \cap Q(L)$ generates $H$ as a $J$-module.

Since $J$ is basic, every element of $H(L)$ can be written as a sum $\sum j_{i} q_{i}$, where the $j_{l}$ 's are in $J$ and the $q_{t}$ 's in $Q(L)$. Now, $Q(L)$ is clearly contained in the right $N$-annihilated part ${ }^{N} H(L)$ of $H(L)$, and repeated 
right-translation by elements of $N$ will annihilate any element of $H(L)$. If the result of the lemma does not hold, then we can find an element $h$ of $H$ which has an expression as $\sum j_{i} q_{i}$, where not all the $q_{i}$ 's are in $H$, and among such elements $h=\sum j_{i} q_{i}$, we can pick one that is of minimal length and such that all of the $j_{l}$ 's lie in the right- $N$-annihilated part ${ }^{N} J$ of $J$. The reason for making such a choice of $h$ is that by [3, Lemma 4.3], ${ }^{N} J=P(L) H(L)^{R}$, which is stable under both left and right translations.

Let $x$ be an element of $U(L)$. Since $H$ is two-sidedly stable, $t_{r}(x) h$ is also an element of $H$. Let $\delta$ denote the comultiplication map, and let $\delta(x)=\sum_{\alpha} x_{\alpha}^{\prime} \otimes x_{\alpha}^{\prime \prime}$. Then,

$$
t_{r}(x) h=\sum_{\alpha, i} t_{r}\left(x_{\alpha}^{\prime}\right)\left(j_{i}\right) q_{i}\left(x_{\alpha}^{\prime \prime}\right) q_{i} .
$$

Thus, multiplying by $j_{1}$, we see that $H$ contains the element

$$
\sum_{\alpha, i} j_{1} t_{r}\left(x_{\alpha}^{\prime}\right)\left(j_{i}\right) q_{i}\left(x_{\alpha}^{\prime \prime}\right) q_{i} \text {. }
$$

$H$ also contains the following, which is a $J$-multiple of $h$

$$
\sum_{\alpha, i} j_{i} t_{r}\left(x_{\alpha}^{\prime}\right)\left(j_{1}\right) q_{1}\left(x_{\alpha}^{\prime \prime}\right) q_{i} \text {. }
$$

Subtracting the second of these from the first, and using the minimality of the length of the expression for $h$, we get

$$
\sum_{\alpha} j_{1} t_{r}\left(x_{\alpha}^{\prime}\right)\left(j_{l}\right) q_{i}\left(x_{\alpha}^{\prime \prime}\right)=\sum_{\alpha} j_{i} t_{r}\left(x_{\alpha}^{\prime}\right)\left(j_{1}\right) q_{1}\left(x_{\alpha}^{\prime \prime}\right) .
$$

If we evaluate each side of the above equation at an element $y$ of $U(L)$, then, denoting $\delta(y)$ by $\Sigma_{\beta} y_{\beta}^{\prime} \otimes y_{\beta}^{\prime \prime}$, we get

$$
\sum_{\beta, \alpha} j_{1}\left(y_{\beta}^{\prime}\right) j_{i}\left(x_{\alpha}^{\prime} y_{\beta}^{\prime \prime}\right) q_{i}\left(x_{\alpha}^{\prime \prime}\right)=\sum_{\beta, \alpha} j_{l}\left(y_{\beta}^{\prime}\right) j_{1}\left(x_{\alpha}^{\prime} y_{\beta}^{\prime \prime}\right) q_{1}\left(x_{\alpha}^{\prime \prime}\right)
$$

The above can be re-written.

$$
\sum_{\beta}\left\{j_{1}\left(y_{\beta}^{\prime}\right) t\left(y_{\beta}^{\prime \prime}\right)\left(j_{i}\right) q_{i}\right\}(x)=\sum_{\beta}\left\{j_{i}\left(y_{\beta}^{\prime}\right) t\left(y_{\beta}^{\prime \prime}\right)\left(j_{1}\right) q_{1}\right\}(x) .
$$

Moreover, the $t(y)(j)$ 's are in $J$ since we have chosen the $j$ 's to be in a two-sidedly stable subspace of $J$. Owing to the freeness of the $q$ 's over $J$, it follows that, for all $y$ in $U(L)$ and all $i>1$,

$$
\sum_{\beta} j_{1}\left(y_{\beta}^{\prime}\right) t\left(y_{\beta}^{\prime \prime}\right)\left(j_{i}\right)=0 \text {. }
$$

Applying this to the element 1 of $U(L)$, we obtain the equation $\left(j_{1} j_{i}\right)(y)$ $=0$, for all $y$ in $U(L)$. This means that $j_{1} j_{i}$ must be the zero function, so that the chosen element $h$ must be just $j_{1} q_{1}$. 
Now, we see that $\left(H q_{1} \cap H\right)$ is a non-zero left Hopf module for $H$, so that, by [8, Thm. 4.1.1], there is a non-zero element $g$ of $\left(H q_{1} \cap H\right)$ such that $\delta(g)=1 \otimes g$. This is possible only if $q_{1}^{-1}$ is in $H$. Since $H$ is closed under the antipode map, this means that $q_{1}$ is in $H$, which establishes the lemma.

LeMMA 2.2. Let $H$ be any sub Hopf algebra of $H(L)$ that separates the elements of $L$. Then, $H$ contains the representative functions of the adjoint representation of $L$.

Proof. The representative functions of the adjoint representation of $L$ lie in the space of representative functions of the adjoint representation of the Lie algebra $L(H)$ of $H$, which clearly are contained in $H$.

LeMMA 2.3. Let $\rho^{R}$ be the restriction map $H(L) \rightarrow H(R)$. Then, $\rho^{R}$ is injective on $Q(L)$, and there is a normal basic subalgebra $J$ of $H(L)$ such that $\rho^{R}(J)$ is a normal basic subalgebra of $H(R)$.

Proof. The first result is clear; the second follows from the constructions in [5] and [6].

For any $L$-module $V$, we denote by $V^{\prime}$ the semisimple L-module associated with $V$, i.e. the direct sum of the simple factor modules in a composition series for $V$. The following result is well known, but, in the absence of a convenient reference, we give a proof here.

Lemma 2.4. For any L-module $V$, the space $\operatorname{Rep}(V)_{s}$ of semisimple representative functions of $V$ is identical with the space $\operatorname{Rep}\left(V^{\prime}\right)$ of representative functions of the associated semisimple L-module.

Proof. For any $L$-modules $U$ and $W$, we say that $U$ is subordinate to $W$ if $U$ is isomorphic to a module obtained from $W$ by a finite sequence of steps each of which is either the selection of a submodule, or the selection of a homomorphic image, or the direct sum of such modules. It is then straightforward to see that, if $U$ is subordinate to $W$ then $\operatorname{Rep}(U)$ is contained in $\operatorname{Rep}(W)$. Further, if $U$ is semisimple and subordinate to $W$ then $U^{\prime}$ is subordinate to $W^{\prime}$.

The space $\operatorname{Rep}(V)$ is a direct sum of copies of homomorphic images of $V$, so is subordinate to $V$. Thus $\operatorname{Rep}(V)_{s}$ is subordinate to $V$, and, thus, to $V^{\prime}$. But $\operatorname{Rep}(V)_{s}$ is a coalgebra, so is its own space of representative 
functions. This shows that $\operatorname{Rep}(V)_{s}$ is contained in $\operatorname{Rep}\left(V^{\prime}\right)$; the inclusion in the other direction is clear.

Let $P$ be a solvable Lie algebra over $K$ and $V$ a semisimple $P$-module. Then the space of representative functions of $V$ is spanned by elements of $Q(P)$ which we call the component functions of the representation. We denote by $A(P)$ the subgroup of $Q(P)$ that is generated by the component functions of the semisimple representation associated with the adjoint representation of $P$. By [7, Lemma 2.1], any $P$-module $U$ is a direct sum of sub $P$-modules $U_{\mu}$ where the $\mu$ 's are equivalence classes of component functions of $U^{\prime}$ modulo the group $A(P)$, and where the component functions of the $P$-module $U_{\mu}^{\prime}$ lie in the class $\mu$.

Definition. Let $L, R$ be as before. An $L$-module $V$ is called an essential $L$-module if the component functions of $V^{\prime}$ as an $R$-module lie in $A(R)$. An element of $H(L)$ is called an essential representative function if it belongs to an essential $L$-module.

THEOREM 2.5. The basic sub Hopf algebra $B(L)$ of $H(L)$ coincides with the Hopf algebra of all essential representative functions of $L$.

Proof. Let $A(R) \#$ denote the inverse image (under the restriction map) in $Q(L)$ of the group $A(R)$. Let $J$ be a normal basic subalgebra of $H(L)$ such that its restriction image $\rho^{R}(J)$ in $H(R)$ is a normal basic subalgebra (Lemma 2.3). We show first that $J[A(R) \#]$ in a Hopf algebra.

Clearly $J[A(R) \#]$ is stable under right-translations. To prove stability under left-translations is equivalent to proving the right-stability of $\eta(J)[A(R) \#]$, where $\eta$ is the antipode map of $H(L)$. We claim first that every left sub $R$-module of $\eta(J)$ is an essential $R$-module. By the result quoted above from [7], it suffices to show that the component function of any one-dimensional sub $R$-moldule of $\eta(J)$ lies in $A(R)$. Let $u \in \eta(J)$ span a one-dimensional sub $R$-module with component function $h$, say. $h$ is the restriction of an element of $H(L)$ and is semisimple; it is easy to see that $h$ must be the restriction of a semisimple element of $H(L)$. Thus, $[L, R]$ annihilates $h$, and there is thus an element, $g$ say, of $Q(L)$ that restricts to $h$. Then, $g^{-1} u$ is in $H(L)^{R}$ which is contained in $\eta(J)$. Since $u$ is chosen to be in $\eta(J)$ this implies that $g=1$. Now, for an element $q$ of $Q(L)$, let $\pi_{q}$ denote the projection from $H(L)$ to $\eta(J) q$. If $x \in L$, then 
$\pi_{q} \circ t_{r}(x)$ is a left $L$-module endomorphism of $H(L)$, and so, maps essential $R$-modules to essential $R$-modules. It is clear then that $\pi_{q} \circ t_{r}(x)(\eta(J))=(0)$ unless $q \in A(R) \#$. Thus, $J[A(R) \#]$ is stable under both left and right translations.

To complete the proof that $J[A(R) \#]$ is a Hopf algebra, we need to prove stability under the antipode $\eta$. Since we already have two-sided stability, it suffices to show that, whenever $J[A(R) \#]$ contains the representative functions of an $L$-module $V$, it also contains the representative functions of the dual module $\mathrm{V}^{\circ}$. If $V$ is an $n$-dimensional module, then the 'interior product' yields an isomorphism of $L$-modules $V^{0} \otimes$ $\wedge^{n} V \cong \wedge^{n-1} V$. The space $\wedge^{n} V$ is one-dimensional, so that its representative functions are the $K$-multiples of a group-like element $q$ of $H(L)$. If the representative functions of $V$ lie in $J[A(R) \#]$, then $q^{-1}$ is in $A(R) \#$, and the representative functions for $V^{\mathrm{o}}$ also lie in $J[A(R) \#]$.

Let $f$ be an essential representative function, and let $T(f)$ be the $L$-module of left-translates of $f$. Let $\operatorname{Rep}(T(f))$ be the space of representative functions for $T(f) ; \operatorname{Rep}(T(f))$ is a finite-dimensional sub coalgebra of $H(L)$. Clearly, there are finite dimensional sub coalgebras $Y_{1}, \ldots, Y_{n}$ of $J[A(R) \#]$ and elements $q_{1}, \ldots, q_{n}$ of $Q(L)$ that are distinct modulo $A(R)$ \# such that $\operatorname{Rep}(T(f))$ is contained in $\sum Y_{i} q_{i}$. Let $\pi_{i}$ be the projection of $\operatorname{Rep}(T(f))$ onto $Y_{i} q_{i}$. Each $\pi_{i}$ commutes with left (or right) translations so that the image of $\pi_{l}$ is contained in $\operatorname{Rep}(T(f))$. Thus, we can assume that $\operatorname{Rep}(T(f))$ is the direct sum of the $Y_{i} q_{l}$ 's and that none of the $Y_{i}$ 's are (0). The semisimple elements of $\operatorname{Rep}(T(f))$ are thus exactly $\Sigma\left(Y_{i}\right)_{s} q_{i}$ (see [3, Lemma 3.3]). Moreover, a non-zero coalgebra has a non-zero simple coalgebra so that none of the $\left(Y_{i}\right)_{s}$ 's are $(0)$. It is also clear that the restriction map $\rho^{R}$ does not annihilate any of the $\left(Y_{i}\right)_{s}$ 's (indeed, since $\left(Y_{i}\right)_{s}$ is stable under translations, evaluation at the element 1 of $U(L)$ is not the zero map).

Now, $J_{s}=H(L)^{R}$, so by [3, Lemma 3.3], $\rho^{R}(J[A(R) \#])=K[A(R)]$. Since the element $f$ above is essential, we must have that $\rho^{R}\left(\operatorname{Rep}(T(f))_{s}\right)$ $\subset K[A(R)]$. From Lemma 2.1, and from the above remarks on the $\left(Y_{i}\right)_{s}$ 's, we see that each $q_{i}$ must be in $A(R) \#$, i.e. that $\operatorname{Rep}(T(f))$ must be contained in $J[A(R) \#]$. Thus, $J[A(R) \#]$ is the Hopf algebra of all essential representative functions of $L$. It remains only to show that $J[A(R) \#]=B(L)$, i.e. that there is no proper sub Hopf algebra of $J[A(R) \#]$ that contains $J$. Since $J$ contains $P(L)$, it separates the elements of $L$ (indeed, a zero of $J$ is thus a zero of $Q(L)$, the exponential image of $P(L)$, and, thus, of all of $H(L))$. By Lemma 2.2, then, $\rho^{R}(B(L))$ must contain $A(R)$, so that, by Lemma $2.1, B(L)$ must contain $A(R) \#$. This completes the proof. 
THEOREM 2.6. Let $L$ be a Lie algebra over $K, R$ the radical of $L$, and let $V$ be an L-module. Let $\left\{q_{1} \#, \ldots, q_{n} \#\right\}$ be the subset of $Q(L)$ whose restriction image in $H(R)$ is the set of component functions of $V^{\prime}$ as an $R$-module. Then the space of representative functions of $V$ is contained in $\sum_{1}^{n} B(L) q_{i} \#$.

Proof. The proof is almost identical with the second part of the proof of Theorem 2.5. In fact, there are finite-dimensional sub coalgebras $Y_{1}, \ldots, Y_{m}$ of $B(L)$, and elements $p_{1}, \ldots, p_{m}$ of $Q(L)$ that are distinct modulo $A(R)$ \# and such that the representative functions of $V$ are contained in $\sum Y_{i} p_{i}$. As in the proof of Theorem 2.5, we find that each $p_{i}$ must be equivalent modulo $A(R) \#$ to one of the $q_{j} \#$ 's. In view of Theorem 2.5, this completes the proof.

Definition. A sub Lie algebra $L_{1}$ of a Lie algebra $L_{2}$ is called an essential subalgebra if the radical of $L_{1}$ is contained in the radical of $L_{2}$ and if every essential representation of $L_{2}$ restricts to an essential representation of $L_{1}$.

LeMma 2.7. A sub Lie algebra $L_{1}$ of a Lie algebra $L_{2}$ is an essential subalgebra if and only if the radical of $L_{1}$ is contained in that of $L_{2}$ and the adjoint representation of $L_{2}$ restricts to an essential representation of $L_{1}$.

\section{(The proof is straightforward.)}

Note. It is an easy consequence of Lemma 2.7 that an ideal in a Lie algebra is an essential subalgebra.

\section{Behavior of $H(L)$ with respect to restriction; the extension results.}

THEOREM 3.1. Let $R_{1}, R_{2}$ be solvable Lie algebras over $K$, let $\phi$ : $R_{1} \rightarrow R_{2}$ be an injective morphism and let $\phi^{*}$ be the induced morphism of Hopf algebras $H\left(R_{2}\right) \rightarrow H\left(R_{1}\right)$. Then the basic sub Hopf algebra $B\left(R_{1}\right)$ of $H\left(R_{1}\right)$ is contained in $\phi^{*}\left(B\left(R_{2}\right)\right)$.

Proof. We prove this in two steps, according to the following two lemmas.

LEMMA 3.2. Let $\phi: L_{1} \rightarrow L_{2}$ be an injection of Lie algebras such that $\phi\left(L_{1}\right)$ is an ideal of $L_{2}$. Then $B\left(L_{1}\right)=\phi^{*}\left(B\left(L_{2}\right)\right)$. 
Proof. It is a consequence of the note at the end of $\S 2$ that $\phi^{*}\left(B\left(L_{2}\right)\right)$ is contained in $B\left(L_{1}\right)$. By a theorem of Zassenhaus (see, for example, [2, Chap. I, §7]), every $L_{1}$-module on which $\left[L_{2}, L_{2}\right] \cap \operatorname{Rad}\left(L_{1}\right)$ acts nilpotently can be embedded in an $L_{2}$-module. In particular, this covers the case of an essential $L_{1}$-module. Thus, $B\left(L_{1}\right)$ is contained in $\phi^{*}\left(H\left(L_{2}\right)\right)$. It is then easy from Theorem 2.5 to show that $B\left(L_{1}\right)$ must actually be contained in $\phi^{*}\left(B\left(L_{2}\right)\right)$.

LemMA 3.3. Let $\phi, R_{1}, R_{2}$ be as in the statement of Theorem 3.1, and let $\psi: R_{2} \rightarrow \operatorname{End}(V)$ be a faithful representation of $R_{2}$. Let $R_{1}^{+}, R_{2}^{+}$be the smallest algebraic subalgebras of $\operatorname{End}(V)$ to contain $\psi\left(\phi\left(R_{1}\right)\right), \psi\left(R_{2}\right)$ respectively, and let $\rho$ be the restriction map from $H\left(R_{2}^{+}\right)$to $H\left(R_{1}^{+}\right)$. Then, $B\left(R_{1}^{+}\right)$is contained in $\rho\left(B\left(R_{2}^{+}\right)\right)$.

Proof. The idea of this lemma is that $R_{1}^{+}$is sufficiently nicely embedded in $R_{2}^{+}$to enable us to construct a normal basic subalgebra $J_{2}$ of $H\left(R_{2}^{+}\right)$such that $\rho\left(J_{2}\right)$ is a normal basic subalgebra of $H\left(R_{1}^{+}\right)$. Specifically, each $R_{i}^{+}$can be written as a semidirect sum of a nilpotent ideal $X_{l}$ (that contains the commutator ideal) and an abelian subalgebra $Y_{\imath}$ in such a way that $X_{1} \subset X_{2}$ and $Y_{1} \subset Y_{2}$. In [6, pp. 610-611], a normal basic subalgebra is constructed starting with an ordered basis of the Lie algebra. If we use the semidirect sum decompositions above for each $R_{l}^{+}$ in choosing the basis of $R_{i}^{+}$, we can construct normal basic subalgebras $J_{i}$ of $H\left(R_{l}^{+}\right)$such that $J_{1}=\rho\left(J_{2}\right)$. The result follows immediately.

Proof of Theorem 3.1. We note that, in the notation of Lemma 3.3, $\psi \circ \phi$ is an injection of $R_{1}$ as an ideal of $R_{1}^{+}$, while $\psi$ is an injection of $R_{2}$ as an ideal of $R_{2}^{+}$. By applying the result of Lemma 3.2 to both of these injections, we obtain Theorem 3.1 from Lemma 3.3.

Let $\phi: S_{1} \rightarrow S_{2}$ be an injection of semisimple Lie algebras over $K$, and let $\phi^{*}: H\left(S_{2}\right) \rightarrow H\left(S_{1}\right)$ be the induced morphism of Hopf algebras. Clearly, $H(S)$ coincides with $B(S)$, and the group $G(S)$ of algebra homomorphisms from $H(S)$ to $K$ is an affine algebraic group. By [4, Chap. XVIII], the Lie algebra of $G(S)$ is $S$. We see, then, that the injection $\phi$ induces a morphism of algebraic groups $\Phi: G\left(S_{1}\right) \rightarrow G\left(S_{2}\right)$ whose kernel, $T$ say, is a finite central subgroup of $G\left(S_{1}\right)$. Now, there are Cartan subalgebras $C_{1}$ of $S_{1}$ and $C_{2}$ of $S_{2}$ such that $\phi\left(C_{1}\right)$ is contained in $C_{2}$. Let $\Lambda_{i}$ be the set of (integral) weights of $S_{l}$ with respect to $C_{i}$ (for $i=1,2$ ). Let $\phi^{\Lambda}$ be the restriction map from $\Lambda_{2}$ to $\Lambda_{1}$. 
THEOREM 3.4. In the above notation, if $V$ is an $S_{1}$-module, then the space of representative functions of $V$ is contained in $\phi^{*}\left(H\left(S_{2}\right)\right)$ iff the weights of $V$ are in $\phi^{\Lambda}\left(\Lambda_{2}\right)$.

Proof. Let $T_{1}, T_{2}$ be the maximal toroids of $G\left(S_{1}\right), G\left(S_{2}\right)$ whose Lie algebras are $C_{1}, C_{2}$ respectively. Since the kernel $T$ of the map $\Phi$ : $G\left(S_{1}\right) \rightarrow G\left(S_{2}\right)$ is finite and central, it is in $T_{1}$ and is, therefore, the kernel of the restriction $\theta: T_{1} \rightarrow T_{2}$ of $\Phi$. For $i=1,2$, let $\chi\left(T_{i}\right)$ be the group of those polynomial characters of $T_{i}$ that occur in restrictions to $T_{i}$ of polynomial representations of $G\left(S_{i}\right)$. Then, $\theta$ induces a map $\theta^{x}$ that sends $\chi\left(T_{2}\right)$ into $\chi\left(T_{1}\right)$. By means of the connection between finite-dimensional $S_{i}$-modules and $G\left(S_{i}\right)$-modules, there is an isomorphism of groups $\chi\left(T_{i}\right) \cong \Lambda_{i}$ that is compatible with the restriction maps $\theta^{\chi}$ and $\phi^{\Lambda}$.

Now, if a weight $\lambda$ of $V$ is in $\phi^{\Lambda}\left(\Lambda_{2}\right)$, then the corresponding character must be in $\theta^{\chi}\left(\chi\left(T_{2}\right)\right)$ and vice versa. Since $T$ is the kernel of the map $\theta$, this means that $T$ must act trivially on the $\lambda$-weight space of $V$. Since $V$ is a sum of weight spaces, $T$ will act trivially on $V$ iff all the weights of $V$ are in $\phi^{\Lambda}\left(\Lambda_{2}\right)$. It is clear from the theory of factor groups that $\phi^{*}\left(H\left(S_{2}\right)\right)$ is the $T$-fixed part of $H\left(S_{1}\right)$, and, thus, that $T$ acts trivially on $V$ iff the representative functions of $V$ are in $\phi^{*}\left(H\left(S_{2}\right)\right)$. This completes the proof.

THEOREM 3.5. Let $\phi: L_{1} \rightarrow L_{2}$ be an injection of Lie algebras over $K$ and let $S_{1}, S_{2}$ be maximal semisimple subalgebras of $L_{1}, L_{2}$ respectively such that $\phi\left(S_{1}\right) \subset S_{2}$. Suppose that $L_{1}=\left[L_{1}, L_{1}\right]$. Then, the representative functions for an $L_{1}$-module $V$ lie in $\phi^{*}\left(H\left(L_{2}\right)\right)$ iff the representative functions of $V$ qua $S_{1}$-module lie in the restriction image $\phi_{S}^{*}\left(H\left(S_{2}\right)\right)$ of $H\left(S_{2}\right)$ in $H\left(S_{1}\right)$.

Proof. By [4, Chap. XVIII], $L_{1}=\left[L_{1}, L_{1}\right]$ iff $H\left(L_{1}\right)$ is finitely generated as an algebra. Moreover, in such a case, the Lie algebra of $H\left(L_{1}\right)$ is $L_{1}$. Let $G\left(L_{1}\right), G\left(L_{2}\right)$ be the pro-affine algebraic groups corresponding to the Hopf algebras $H\left(L_{1}\right), H\left(L_{2}\right)$ respectively, and let $\Phi$ be the induced morphism $G\left(L_{1}\right) \rightarrow G\left(L_{2}\right)$. As in the proof of Theorem 3.4, the kernel, $T$ say, of $\Phi$ is a finite central subgroup, and is thus contained in every maximal linearly reductive subgroup of $G\left(L_{1}\right)$.

Let $G\left(L_{1}\right)=G_{u} \cdot P$ be a decomposition of $G\left(L_{1}\right)$ as a semidirect product of its unipotent radical $G_{u}$ and a maximal linearly reductive subgroup $P$. Since every (finite-dimensional) $L_{1}$-module is a $G\left(L_{1}\right)$-module, the Lie algebra of $G_{u}$ is the intersection of the kernels of all semisimple $L_{1}$-modules. In the case where $L_{1}=\left[L_{1}, L_{1}\right]$, this is the 
radical of $L_{1}$. Consequently, the (linearly reductive) subgroups corresponding to maximal semisimple subalgebras of $L_{1}$ are maximal linearly reductive subgroups. By the conjugacy of such subgroups, we see that we may suppose that the Lie algebra of $P$ is $S_{1}$.

The injection $\phi_{S}: S_{1} \rightarrow S_{2}$ induces a morphism of algebraic groups $\Phi_{S}: G\left(S_{1}\right) \rightarrow G\left(S_{2}\right)$, where the $G(S)$ 's are as in Theorem 3.4. Let $T_{S}$ be the kernel of $\Phi_{S}$. Now, the injection of $S_{1}$ into $L_{1}$ induces an injection of $G\left(S_{1}\right)$ into $G\left(L_{1}\right)$, and similarly for $S_{2}$ (as follows from the fact that every $S_{i}$-module can be regarded as an $L_{i}$-module). The image of $G\left(S_{1}\right)$ in $G\left(L_{1}\right)$ is clearly $P$, and the map $G\left(S_{1}\right) \rightarrow P$ is an isomorphism. Now, we need only note that $T$ is the kernel of the map (the restriction of $\Phi$ ) from $P$ to $G\left(L_{2}\right)$, while $T_{S}$ is the kernel of the map from $G\left(S_{1}\right)$ to $G\left(S_{2}\right) \subset$ $G\left(L_{2}\right)$. Therefore the isomorphism from $G\left(S_{1}\right)$ to $P$ maps $T_{S}$ onto $T$. Since $\phi^{*}\left(H\left(L_{2}\right)\right)$ is the $T$-fixed part of $H\left(L_{1}\right)$, and $\phi_{S}^{*}\left(H\left(S_{2}\right)\right)$ the $T_{S}$-fixed part of $H\left(S_{1}\right)$, the result of the theorem now follows.

We are now in a position to prove the extension theorem for representations of Lie algebras that was mentioned at the beginning.

Let $L_{1}$ be a subalgebra of a Lie algebra $L_{2}$ over $K$, and let $R_{1}$ and $R_{2}$ be the radicals of $L_{1}$ and $L_{2}$. Let $S_{1}$ and ${ }_{2}$ be maximal semisimple subalgebras of $L_{1}$ and $L_{2}$ such that $S_{1}$ is contained in $S_{2}$. Let $C_{1}$ and $C_{2}$ be Cartan subalgebras of $S_{1}$ and $S_{2}$ such that $C_{1}$ is contained in $C_{2}$. Let $V$ be a finite-dimensional $L_{1}$-module.

THEOREM 3.6. In the above notation, assume that either (a) $R_{1}$ is contained in $R_{2}$ or (b) $L_{1}=\left[L_{1}, L_{1}\right]$. Then $V$ can be embedded as a sub $L_{1}$-module in a finite dimensional $L_{2}$-module iff both (i) $\left[L_{2}, L_{2}\right] \cap R_{1}$ acts nilpotently on $V$ and (ii) the weights for $V$ as a $C_{1}$-module are restrictions of integral weights for $C_{2}$.

Proof. Condition (ii) is evidently necessary in all cases. In case (a), $\left[L_{1}, L_{2}\right] \cap R_{1}$ is contained in $\left[L_{2}, R_{2}\right]$ which acts nilpotently on any $L_{2}$-module, while, in case (b), all of $R_{1}$ necessarily acts nilpotently on an $L_{1}$-module. Thus, in both cases, conditions (i) and (ii) are necessary.

The sufficiency in case (b) is a consequence of Theorems 1.3, 3.4, and 3.5. We may restrict ourselves, then, to case (a).

The Levi decompositions (suppressing the indices 1 and 2) $L=R+S$ induce isomorphisms of algebras from $H(L)^{R} \otimes{ }^{S} H(L)$ to $H(L)$, where $H(L)^{R}$ denotes the subspaces of $H(L)$ that is annihilated by left-translations by elements of $R$, and ${ }^{S} H(L)$ the subspace annihilated by righttranslations by elements of $S$ (see [4, XVIII.4]). We need to make the 
isomorphisms explicit. Let $\rho^{R}: H(L) \rightarrow H(R)$ and $\rho^{S}: H(L) \rightarrow H(S)$ be the restrictions; we note that $\rho^{S}$ is surjective. The restriction maps are pre-inverted by algebra isomorphisms $j^{R}: \rho^{R}(H(L)) \rightarrow{ }^{S} H(L)$ and $j^{S}$ : $H(S) \rightarrow H(L)^{R}$. If $\delta$ is the comultiplication and $\mu$ the multiplication on $H(L)$, then $\mu \circ\left\{\left(j^{S} \circ \rho^{S}\right) \otimes\left(j^{R} \circ \rho^{R}\right)\right\} \circ \delta$ is the identity map on $H(L)$.

Let $\phi$ denote the injection of $L_{1}$ into $L_{2}, \phi_{R}$ that of $R_{1}$ into $R_{2}$ and $\phi_{S}$ that of $S_{1}$ into $S_{2}$. By Theorem 2.6 the space of representative functions of $V$ qua $R_{1}$-module is contained in $\sum B\left(R_{1}\right) q_{i}$, where the $q_{i}$ 's are the component functions of the associated semisimple $R$-module $V^{\prime}$. By Theorem 3.1, $B\left(R_{1}\right)$ is contained in $\phi_{R}^{*}\left(B\left(R_{2}\right)\right)$, while, by Lemma 3.2, $B\left(R_{2}\right)=\rho^{R}\left(B\left(L_{2}\right)\right)$. If condition (i) holds, then the restriction to $R_{1}$ of each component function $q_{i}$ is a Lie algebra homomorphism $R_{1} \rightarrow K$ that annihilates $\left[L_{2}, L_{2}\right] \cap R_{1}$ and, thus, extends to a Lie algebra homomorphism $L_{2} \rightarrow K$. This implies that each $q_{i}$ is in $\phi_{R}^{*}\left(\rho^{R}\left(H\left(L_{2}\right)\right)\right)$, whence all of the representative functions of $V$ as an $R_{1}$-module lie in $\phi_{R}^{*}\left(\rho^{R}\left(H\left(L_{2}\right)\right)\right)$. By Theorem 3.4, condition (ii) implies that the representative functions of $V$ as an $S_{1}$-module lie in $\phi_{S}^{*}\left(H\left(S_{2}\right)\right)$.

To complete the proof, we remark that the algebra homomorphism $j^{R}: \rho^{R}\left(H\left(L_{1}\right)\right) \rightarrow{ }^{S}\left(H\left(L_{1}\right)\right)$ maps $\phi_{R}^{*}\left(\rho^{R}\left(H\left(L_{2}\right)\right)\right)$ into $\phi^{*}\left({ }^{S} H\left(L_{2}\right)\right)$; similarly, $j^{S}$ maps $\phi_{S}^{*}\left(H\left(S_{2}\right)\right)$ into $\phi^{*}\left(H\left(L_{2}\right)^{R}\right)$. We now apply the map $\mu \circ\left\{\left(j^{S} \circ \rho^{S}\right) \otimes\left(j^{R} \circ \rho^{R}\right)\right\} \circ \delta$ to the space of representative functions of $V$. Since the space of representative functions is a sub-coalgebra, $\delta$ sends it into its tensor square. Now $\rho^{R}$ maps the space of representative functions of $V$ into the space of representative functions of $V$ qua $R_{1}$-module; similarly for $\rho^{S}$. It is clear from the above, then, that $\mu \circ\left\{\left(j^{S} \circ \rho^{S}\right) \otimes\left(j^{R} \circ \rho^{R}\right)\right\} \circ \delta$ maps the space of representative functions of $V$ into $\phi^{*}\left(H\left(L_{2}\right)\right)$. This map is, however, the identity, so, by Theorem 1.2 , the proof is complete.

\section{REFERENCES}

[1] A. Bialynicki-Birula, G. Hochschild, and G. D. Mostow, Extensions of representations of algebraic linear groups, Amer. J. Math., 85 (1963), 131-144.

[2] N. Bourbaki, Elements de Mathematique, Groupes et Algebres de Lie, Hermann, 1960.

[3] W. Giles, On the algebra of representative functions of a lie algebra, Trans. Amer. Math. Soc., 109 (1963), 101-120.

[4] G. Hochschild, Basic Theory of Algebraic Groups and Lie Algebras, Springer Verlag, GTM 75, 1981.

[5] _ Algebraic Lie algebras and representative functions, Illinois J. Math., 3 no. 4 (1959), 499-523. 
[6] Algebraic Lie algebras and representative functions, Supplement, Illinois J. Math., 4 no. 4, (1960), 609-618.

[7] G. Hochschild and G. D. Mostow, On the algebra of representative functions of an analytic group II, Amer. J. Math., 86, no. 4 (1964), 869-887.

[8] M. Sweedler, Hopf Algebras, Benjamin, 1969.

Received November 15, 1984.

University of California

BERKELEY, CA 94720 


\section{PACIFIC JOURNAL OF MATHEMATICS EDITORS}

\author{
V. S. VARADARAJAN \\ (Managing Editor) \\ University of California \\ Los Angeles, CA 90024 \\ Herbert Clemens \\ University of Utah \\ Salt Lake City, UT 84112 \\ R. FINN \\ Stanford University \\ Stanford, CA 94305
}

\author{
HERMANN FLASCHKA \\ University of Arizona \\ Tucson, AZ 85721 \\ RAMESh A. GANGOLli \\ University of Washington \\ Seattle, WA 98195 \\ VAUGHAN F. R. JONES \\ University of California \\ Berkeley, CA 94720 \\ ROBION KIRBY \\ University of California \\ Berkeley, CA 94720 \\ C. C. MOORE \\ University of California \\ Berkeley, CA 94720 \\ H. SAMELSON \\ Stanford University \\ Stanford, CA 94305 \\ HAROLD STARK \\ University of California, San Diego \\ La Jolla, CA 92093
}

\section{ASSOCIATE EDITORS}
R. ARENS
E. F. BECKENBACH
B. H. NEUMANN
F. WOLF
K. YOSHIDA (1906-1982)

\section{SUPPORTING INSTITUTIONS}
UNIVERSITY OF ARIZONA
UNIVERSITY OF BRITISH COLUMBIA
CALIFORNIA INSTITUTE OF TECHNOLOGY
UNIVERSITY OF CALIFORNIA
MONTANA STATE UNIVERSITY
UNIVERSITY OF NEVADA, RENO
NEW MEXICO STATE UNIVERSITY
OREGON STATE UNIVERSITY
UNIVERSITY OF OREGON UNIVERSITY OF SOUTHERN CALIFORNIA STANFORD UNIVERSITY UNIVERSITY OF HAWAII UNIVERSITY OF TOKYO UNIVERSITY OF UTAH WASHINGTON STATE UNIVERSITY UNIVERSITY OF WASHINGTON 


\section{Pacific Journal of Mathematics \\ Vol. 127, No. $1 \quad$ January, 1987}

Jacob Burbea, Boundary behavior of holomorphic functions in the ball ..... 1

Jan Dijkstra, Strong negligibility of $\sigma$-compacta does not characterize

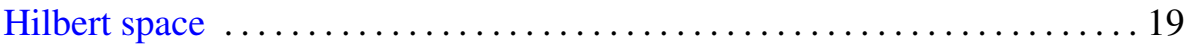

Ruy Exel, Rotation numbers for automorphisms of $C^{*}$ algebras $\ldots \ldots \ldots 31$

Howard Jacobowitz, The canonical bundle and realizable CR

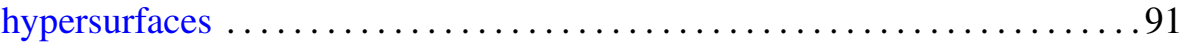

James T. Joichi and Dennis Warren Stanton, Bijective proofs of basic

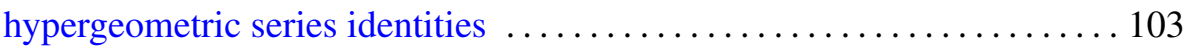

Gareth J. Knowles, Quotients of nest algebras with trivial commutator .... 121

Murray Angus Marshall, Exponentials and logarithms on Witt rings ..... 127

Courtney Hughes Moen, The dual pair $(U(3), U(1))$ over a $p$-adic field . ...141

William Ortmeyer, Surgery on a class of pretzel knots $\ldots \ldots \ldots \ldots \ldots \ldots 155$

John Gerard Ryan, Extensions of representations of Lie algebras . . . . . 173

Ivan Charles Sterling, A generalization of a theorem of Delaunay to

rotational $W$-hypersurfaces of $\sigma_{l}$-type in $H^{n+1}$ and $S^{n+1} \ldots \ldots \ldots 187$

Vesko M. Valov, Another characterization of AE(0)-spaces $\ldots \ldots \ldots \ldots \ldots 9$ 